\title{
CONDIÇÕEs HidrológICAS E RESUltados de CULTIVO EXPERIMENTAL DO CAMARÃO MARINHO Litopenaeus Vannamei (BOONE, 1931), EM VIVEIROS ESTUARINOS DA ILHA DE ITAMARACÁ (PERnAMbuCO-BRASIL).
}

\author{
LOURINALDO BARRETO CAVALCANTI ${ }^{1}$ \\ SÍlVIO JOSÉ DE MACEDO ${ }^{2}$ \\ PATRÍCIA FERNANDES DE CASTRO ${ }^{1}$ \\ MÁrCio FranCiSCO ALVES DE SANTANA ${ }^{2}$ \\ 1 Empresa Pernambucana de Pesquisa \\ Agropecuária - IPA \\ 2 Universidade Federal de Pernambuco - \\ Departamento de Oceanografia
}

\begin{abstract}
RESUMO
No presente trabalho, foram analisadas as variações dos parâmetros físicos e químicos da água de dois viveiros naturais, com área individual de $4.200 \mathrm{~m}^{2}$, utilizados para cultivo experimental do camarão marinho Litopenaeus vannamei, na Base de Piscicultura de Itamaracá. O período de cultivo estendeu-se de 6 de novembro de 1999 a 3 de fevereiro de 2000 (90 dias), usando-se densidades de povoamento de 5,95 e $3,57 \mathrm{PL} / \mathrm{s} / \mathrm{m}^{2}$, sendo que apenas um dos viveiros recebeu ração industrializada como complemento alimentar para os camarões. Foram efetuadas coletas mensais de amostras de água, em dias coincidentes com as biometrias quinzenais e também uma variação nictemeral, para medição de temperatura, transparência, $\mathrm{pH}$, salinidade, oxigênio dissolvido, nitrito, nitrato, fosfato e silicato. Os valores dessas variáveis oscilaram dentro de limites satisfatórios ao desenvolvimento dos camarões, que alcançaram peso médio de 16$17 \mathrm{~g}$, ao final dos 90 dias de cultivo, com produtividades variando de 310 a 450 $\mathrm{Kg} / \mathrm{ha} /$ ciclo.
\end{abstract}

Palavras chave: hidrologia, camarão, cultivo.

\begin{abstract}
This paper evaluate the physical and chemical variations of the water of two earthen ponds, with an area of $4,200 \mathrm{~m}^{2}$ each, which were used for the experimental cualtivation of the marine shrimp Litopenaeus vannamei, in the fishculture base of Itamaracá. The period of cultivation was from November $6^{\text {th }}$ 1999 to February $3^{\text {th }} 2000$ (90 days), during which time the stocking densities of
\end{abstract}

Trab. Oceanog. Univ. Fed. PE, Recife, v 28, n. 2, 2000. p. 209-222 
5.95 and 3.57 PL's $/ \mathrm{m}^{2}$ were used. Only one of the ponds received industrialized ration as a food supplement for the shrimp. Water samples were taken monthly in days coinciding with the biweekly biometry, and also a nyctemeral variation was carried out to measure temperature, transparency, $\mathrm{pH}$, salinity, dissolved oxygen, nitrite, nitrate, phosphate and silicate. The values of these variables ranged within satisfactory limits of shrimp development, which reached and average weight of $16-17 \mathrm{~g}$ at the $\mathrm{d}$ of 90 days of cultivation. Productivity varied from 310 to 450 $\mathrm{kg} / \mathrm{ha} /$ cycle.

Key words: Hydrology, shrimp cultivation.

\section{INTRODUÇÃO}

A limitação dos estoques naturais de camarão e o desenvolvimento de novas tecnologias têm levado a carcinicultura marinha a um desenvolvimento acelerado em todo o mundo. No Brasil, ela vem crescendo com taxas acima de $100 \%$ ao ano, fazendo prever que o país tornar-se-á, a médio prazo, um dos líderes mundiais do setor.

Uma das espécies cultivadas é o Litopenaeus vannamei (Boone, 1931), originária do Oceano Pacífico e introduzida em nosso país há cerca de uma década. Participa da quase totalidade dos cultivos, conduzidos em sistema semi-intensivo, alcançando no presente, produtividades médias em torno de $4.000 \mathrm{Kg} / \mathrm{ha} / \mathrm{ano}$.

A expansão rápida da atividade, no entanto, tem dado motivo a questionamentos quanto a possíveis danos ambientais que possam ser causados pelo manejo inadequado dos cultivos, principalmente no que diz respeito à qualidade dos efluentes que são drenados para os ambientes adjacentes aos viveiros.

Segundo Boyd (1989), mesmo considerando as modernas técnicas atualmente utilizadas nas fazendas de cultivo de camarões marinhos, a produção poderá, em muitos casos, ser afetada pela degradação da qualidade da água.

Por outro lado, dependendo do sistema de cultivo e do manejo adotado durante a fase de crescimento do camarão, a água pode ter as suas características físicas e químicas substancialmente alteradas, com reflexos negativos no ambiente adjacente, em razão da drenagem dos efluentes.

Assim sendo, o conhecimento e controle das características físicas e químicas da água dos ambientes de cultivo tem grande importância, quando se considera a necessidade de se praticar a atividade de carcinicultura, de acordo com os preceitos básicos de sustentabilidade.

Diante disto, procurou-se, através da pesquisa realizada, interpretar as variações dos parâmetros físicos e químicos da água em dois viveiros estuarinos localizados na Base de Piscicultura de Itamaracá, onde foram conduzidos cultivos experimentais de L. vannamei.

Trab. Oceanog. Univ. Fed. PE, Recife, v 28, n. 2, 2000. p. 209-222 


\section{DESCRIÇÃO DA ÁREA}

A Base fica localizada às margens do Canal de Santa Cruz, em uma área pertencente ao governo do Estado de Pernambuco, que opera a mesma em parceria com o Departamento de Oceanografia da Universidade Federal de Pernambuco, para realização de pesquisas e produção de peixes estuarinos e camarões marinhos (Figura 1). Dispõe de 27 viveiros naturais com tamanhos variados, que são abastecidos, na sua maioria, por um canal oriundo do Canal de Sta. Cruz, havendo renovação de água, apenas durante as preamares de sizígia e assim mesmo, de forma bastante precária. $\mathrm{O}$ abastecimento é feito através de comportas de alvenaria de vários tipos, com sistema de tábuas móveis, que permitem regular a entrada e saída de água, de acordo com a altura da maré.

A área é caracterizada por sedimentos holocênicos, que constituem os mangues e aluviões da Ilha de Itamaracá. Este contato e a declividade do terreno da área têm caráter benéfico para os sedimentos dos viveiros, por enriquecê-los com carbonato de cálcio (Macedo et al.,1980).

\section{MATERIAL E MÉTODOS}

Para o experimento de engorda, foram usados dois viveiros (Viv. 2 e Viv. 3 ), com área individual de $4.200 \mathrm{~m}^{2}$, profundidade média de $0,80 \mathrm{~m}$ e com abastecimento feito através de uma camboa construída no manguezal.

A estocagem dos viveiros efetuou-se com pós-larvas de L. vannamei, com idade de 10dias $\left(\mathrm{PL}_{10}\right)$, adquiridas em um laboratório particular. O Viv. 2 recebeu 25.000 PL's $\left(5,95 / \mathrm{m}^{2}\right)$ e o Viv. 3, 15.000 PL's $\left(3,57 / \mathrm{m}^{2}\right)$, estendendo-se o cultivo, por 90 dias, entre 6 de novembro de 1999 e 3 de fevereiro de 2000. Passado o primeiro mês foram efetuadas biometrias quinzenais usando-se cerca de $10 \%$ da população de camarões.

Os camarões do Viv.2 foram alimentados diariamente com ração industrializada contendo $35 \%$ de proteína, distribuída em comedouros fixos (bandejas). $\mathrm{O}$ arraçoamento teve início um mês após o povoamento, em quantidade diária equivalente a $3 \%$ da biomassa dos camarões. No Viv. 3, os camarões foram cultivados apenas com o alimento natural disponível no próprio ambiente.

As amostragens de água para as análises físicas e químicas realizaram-se nas mesmas datas das biometrias, sempre no período matinal. No dia 25 de janeiro de 2000 foi realizada uma variação nictemeral em ambos os viveiros. No momento das coletas efetuaram-se as medições de temperatura e transparência da água, enquanto que, a partir das amostras obtidas, foram medidos em laboratório os teores de salinidade, $\mathrm{pH}$, oxigênio dissolvido, nitrito, nitrato, fosfato e silicato.

A temperatura da água foi obtida através de termômetro de mercúrio com escala de 0 a $100^{\circ} \mathrm{C}$, a transparência pelo disco de Secchi e o $\mathrm{pH}$, por um potenciômetro Metronal E-120.

Trab. Oceanog. Univ. Fed. PE, Recife, v 28, n. 2, 2000. p. 209-222 
As dosagens de salinidade foram efetuadas pelo método de MohrKnudsen, descrito por Strickland \& Parsons (1972) e o oxigênio dissolvido pelo método de Winkler, modificado por Strickland \& Parsons (1965).

Para as análises de nitrito, nitrato e fosfato, empregou-se o método de Strickland \& Parsons (1972), e nas de silicato, o método de Grasshoff et al. (1983).

\section{RESULTADOS E DISCUSSÃO}

Os valores e as variações dos parâmetros físicos e químicos, medidos durante o ciclo de cultivo nos dois viveiros selecionados para a pesquisa, são apresentados nas Tabelas de 1 a 4 e nas Figuras de 2 a 5.

Os índices de transparência da água variaram sempre entre 40 e $70 \mathrm{~cm}$, em ambos os viveiros, de certa forma semelhantes aos encontrados por SipaúbaTavares (1998), que considerou a faixa de 45 a $60 \mathrm{~cm}$, como adequada para cultivo.

Os valores de temperatura, no curso do experimento situaram-se em torno de $30^{\circ} \mathrm{C}$, caracterizando bem a época seca na região. Valores semelhantes foram assinalados por Macedo et al. (1980), na mesma área. Os valores encontrados estão dentro da faixa ideal para cultivo do L. vannamei, pois Guerrero-Galvan (1999), analisando dados de cultivo desse camarão em viveiros da costa do México, detectou a faixa de 25 a $32^{\circ} \mathrm{C}$, como a de melhor crescimento.

Segundo Boyd (1990), existem poucas informações sobre os efeitos do $\mathrm{pH}$ no metabolismo dos camarões, mas pode-se afirmar que respondem às variações deste parâmetro, da mesma forma que os peixes, os quais crescem satisfatoriamente numa faixa de 6 a 9 . No caso em estudo, o pH apresentou pequenas oscilações entre 8 e 9, sendo que os valores medidos nas coletas de dezembro foram ligeiramente superiores aos encontrados por ocasião da variação nictemeral, na fase final do cultivo.

Quanto à salinidade, as várias espécies de camarões marinhos necessitam de faixas específicas para crescer. O Litopenaeus stylirostris, por exemplo, sobrevive bem e cresce normalmente entre 15 e $45 \%$, podendo até tolerar níveis mais elevados (Clifford, 1998). No caso particular do L. vannamei, o mesmo pode ser cultivado em salinidades variando de 0 a $40 \%$, sendo que a faixa de 20 a $25 \%$ o é considerada como ideal. No presente estudo, foram encontrados, no mês de dezembro, valores superiores a $40 \%$ em ambos os viveiros, em decorrência provavelmente da não renovação de água, posto que as coletas coincidiram com marés de quadratura. Por outro lado, os valores registrados no Viv. 2, durante a variação nictemeral, foram menores que os do Viv.3, indicando que a renovação, no primeiro, se processou de forma mais eficiente.

O oxigênio dissolvido é uma das variáveis químicas mais importantes em um ambiente de cultivo, pois as suas concentrações influem diretamente na vida dos animais. Segundo Boyd (1990), baixos níveis provocam redução no crescimento e uma susceptibilidade a doenças. Tratando-se de camarões marinhos, Bedier\&Soyig (1988) comprovaram que a sobrevivência e o crescimento de

Trab. Oceanog. Univ. Fed. PE, Recife, v 28, n. 2, 2000. p. 209-222 
juvenis e adultos de L. vannamei decresceram a uma concentração constante de $1,05 \mathrm{ml} / \mathrm{L}$ Por outro lado, Hopkins et al. (1991) constataram que o oxigênio, em um nível de $0,70 \mathrm{ml} / \mathrm{L}$ pode ser letal. Durante todo o período de cultivo, essa variável situou-se dentro de limites considerados satisfatórios para a sobrevivência e crescimento dos camarões. Por ocasião da variação nictemeral, observou-se, nos dois viveiros, índices de supersaturação no período diurno indicando grande atividade fotossintética.

Com relação aos nutrientes, segundo Boyd (1990), um grande número de elementos inorgânicos é requerido para o crescimento do fitoplâncton. $\mathrm{O}$ nitrogênio e o fósforo são os dois elementos que, provavelmente, mais condicionam esse crescimento e, consequentemente, mais contribuem para o aumento da produtividade primária. No caso da sílica, a sua importância é mais diretamente ligada ao desenvolvimento das diatomáceas. No decorrer da pesquisa, o nitrito apresentou concentrações relativamente baixas, tendo-se observado apenas um valor de $0,833 \mu \mathrm{mol} / \mathrm{L}$, no Viv.3, durante a variação nictemeral. Com relação ao nitrato, as variações foram significativas, observando-se em dezembro, valores superiores a $3,0 \mu \mathrm{mol} / \mathrm{L}$, em ambos os viveiros. O fosfato oscilou entre $0,631 \mathrm{e}$ $2,355 \mu \mathrm{mol} / \mathrm{L}$, sendo mais elevado sempre no Viv.3 e o silicato apresentou a mesma tendência, tendo sido registrado um valor máximo de $59,704 \mu \mathrm{mol} / \mathrm{L}$ em dezembro.Os maiores valores registrados no Viv.3 podem indicar que houve uma maior degradação de matéria orgânica no ambiente, acumulada em conseqüência da renovação de água deficiente. Os resultados obtidos são bastante próximos aos encontrados por Macedo et al. (1980), em viveiros de cultivo de peixe, na mesma área.

Os dados de crescimento obtidos durante as biometrias e a variação do peso médio dos camarões ao longo do tempo de cultivo podem ser vistos na Tabela 5 e Figura 6, respectivamente. O peso médio final dos camarões nos dois viveiros situou-se entre 16 e $17 \mathrm{~g}$, resultado que pode ser considerado bom quando comparado aos obtidos em cultivos comerciais, nos quais o peso médio na época da despesca é de 10-12g, após 75-90 dias de cultivo (Cavalcanti et al., 2000; Castro et al., 2000).

Neste trabalho, ao fim do cultivo, foram despescados $192 \mathrm{~kg}$ de camarão no Viv.2 e $130 \mathrm{~kg}$ no Viv.3, correspondendo a produtividades médias de $457 \mathrm{e}$ $310 \mathrm{~kg} / \mathrm{ha} / \mathrm{ciclo}$, respectivamente. Esses valores situam-se dentro das faixas alcançadas no início da história do camarão marinho no Brasil, marcado pelo cultivo em densidades baixas ( 2 a 3 indivíduos $/ \mathrm{m}^{2}$ ), onde eram obtidas produtividades de 300-600kg/ha/ano (Maia, 1993; Maia et al.,2000; Nunes,2000).

As taxas de sobrevivência observadas no presente experimento foram inferiores às normalmente obtidas em cultivos comerciais, mesmo considerando-se que as densidades de povoamento foram baixas $\left(5,95\right.$ e $3,57 \mathrm{PL} / \mathrm{s} / \mathrm{m}^{2}$ nos viveiros 2 e 3, respectivamente). Como não houve anormalidades com relação aos parâmetros físicos e químicos da água de cultivo, pode-se atribuir esse fato à ação de peixes predadores, uma vez que foi constatada a presença de alguns exemplares de bicuda

Trab. Oceanog. Univ. Fed. PE, Recife, v 28, n. 2, 2000. p. 209-222 


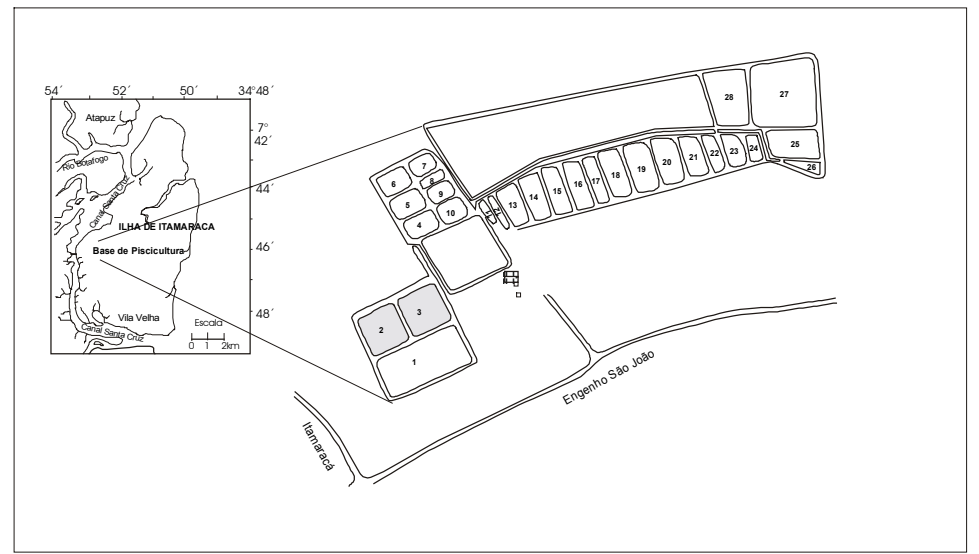

Figura 1 - Viveiros da Base de Piscicultura de Itamaracá-PE.

(Sphyraena barracuda) e ubarana (Elops saurus) nos viveiros, por ocasião da despesca.

Os resultados da pesquisa indicam que, mesmo com a renovação de água deficiente, os viveiros pesquisados ofereceram condições ambientais favoráveis ao desenvolvimento dos camarões. Isto indica, que o $L$. vannamei, por sua rusticidade, pode ser cultivado no âmbito de comunidades litorâneas, em regime extensivo, com baixo custo, oferecendo alternativas de renda complementar aos seus componentes.

Tabela 1 - Dados hidrológicos do Viveiro 2.

\begin{tabular}{|c|c|c|c|c|c|c|c|c|c|c|c|}
\hline Data & Hora & $\begin{array}{c}\text { Secchi } \\
\text { (m) }\end{array}$ & pH & $\begin{array}{c}\mathbf{T} \\
\left({ }^{\circ} \mathbf{C}\right)\end{array}$ & $\begin{array}{c}S \\
(\%)\end{array}$ & 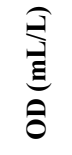 & $\begin{array}{l}\text { OD } \\
(\%)\end{array}$ & 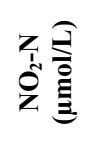 & 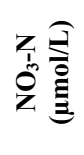 & 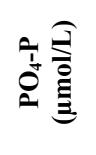 & 응 \\
\hline $14 / 12 / 1999$ & 09:30 & 0,4 & 8,78 & 29,0 & 42,78 & 4,01 & 94,42 & 0,001 & 0,802 & 1,420 & 42,723 \\
\hline 28/12/1999 & 09:30 & 0,4 & 8,37 & 28,9 & 37,43 & 4,00 & 91,29 & 0,136 & 3,547 & 1,008 & 13,361 \\
\hline $11 / 01 / 2000$ & 09:30 & 0,6 & 8,48 & 29,0 & 32,89 & 4,35 & 96,97 & 0,001 & 0,559 & 0,631 & 22,822 \\
\hline
\end{tabular}

Tabela 2 - Dados hidrológicos do Viveiro 3.

\begin{tabular}{|c|c|c|c|c|c|c|c|c|c|c|c|}
\hline Data & Hora & $\begin{array}{c}\text { Secchi } \\
\text { (m) }\end{array}$ & pH & $\begin{array}{c}T \\
\left({ }^{\circ} \mathrm{C}\right)\end{array}$ & $\begin{array}{c}\mathrm{S} \\
(\% 0)\end{array}$ & $\underset{(\mathrm{mL} / \mathrm{L})}{\text { OD }}$ & $\begin{array}{l}\text { OD } \\
(\%)\end{array}$ & 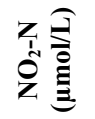 & 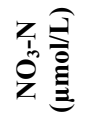 & 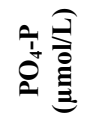 & 象高 \\
\hline $4 / 12 / 99$ & 09:45 & 0,3 & 8,24 & 28,0 & 45,99 & 2,58 & 60,87 & 0,068 & 1,123 & 2,355 & 48,572 \\
\hline
\end{tabular}

Trab. Oceanog. Univ. Fed. PE, Recife, v 28, n. 2, 2000. p. 209-222 
Tabela 3 - Dados hidrológicos nictemerais do Viveiro $2(25 / 01 / 2000)$.

\begin{tabular}{ccccccccccc}
\hline Hora & $\begin{array}{c}\text { Secchi } \\
(\mathbf{m})\end{array}$ & $\mathbf{p H}$ & $\begin{array}{c}\mathbf{T} \\
\left({ }^{\mathbf{}} \mathbf{C}\right)\end{array}$ & $\begin{array}{c}\mathbf{S} \\
(\mathbf{\% o})\end{array}$ & $\begin{array}{c}\mathbf{O D} \\
(\mathbf{m L} / \mathbf{L})\end{array}$ & $\begin{array}{c}\mathbf{O D} \\
(\mathbf{\%})\end{array}$ & $\begin{array}{c}\mathbf{N O}_{2}-\mathbf{N} \\
(\boldsymbol{\mu m o l} / \mathbf{L})\end{array}$ & $\begin{array}{c}\mathbf{N O}_{3}-\mathbf{N} \\
(\boldsymbol{\mu m o l} / \mathbf{L})\end{array}$ & $\begin{array}{c}\mathbf{P O}_{\mathbf{4}}-\mathbf{P} \\
(\boldsymbol{\mu m o l} / \mathbf{L})\end{array}$ & $\begin{array}{c}\mathbf{S i O}_{\mathbf{2}} \\
(\boldsymbol{\mu m o l} / \mathbf{L})\end{array}$ \\
\hline $\mathbf{9 : 0 0}$ & 0,7 & 8,05 & 29,0 & 35,02 & 3,88 & 87,52 & 0,063 & 0,201 & 0,422 & 21,366 \\
$\mathbf{1 2 : 0 0}$ & 0,7 & 8,46 & 30,1 & 35,02 & 5,07 & 116,39 & 0,057 & 0,478 & 0,306 & 27,773 \\
$\mathbf{1 5 : 0 0}$ & 0,7 & 8,04 & 30,2 & 34,76 & 6,88 & 157,97 & 0,001 & 0,001 & 0,393 & 16,602 \\
$\mathbf{1 8 : 0 0}$ & 0,6 & 8,04 & 30,2 & 37,76 & 7,05 & 164,56 & 0,075 & 0,041 & 0,153 & 28,867 \\
$\mathbf{2 1 : 0 0}$ & & 8,33 & 30,0 & 35,02 & 5,74 & 131,56 & 0,034 & 0,548 & 0,275 & 25,961 \\
$\mathbf{0 : 0 0}$ & & 8,03 & 30,0 & 35,02 & 4,64 & 106,35 & 0,136 & 0,014 & 0,300 & 22,987 \\
$\mathbf{3 : 0 0}$ & & 8,14 & 29,0 & 35,02 & 4,09 & 92,25 & 0,078 & 0,798 & 0,474 & 26,498 \\
$\mathbf{6 : 0 0}$ & & 8,22 & 28,0 & 35,02 & 3,32 & 73,68 & 0,014 & 0,037 & 0,431 & 29,542 \\
\hline
\end{tabular}

Tabela 4 - Dados hidrológicos nictemerais do Viveiro 3 (25/01/2000).

\begin{tabular}{ccccccccccc}
\hline Hora & $\begin{array}{c}\text { Secchi } \\
(\mathbf{m})\end{array}$ & $\mathbf{p H}$ & $\begin{array}{c}\mathbf{T} \\
\left({ }^{\mathbf{}} \mathbf{C}\right)\end{array}$ & $\begin{array}{c}\mathbf{S} \\
(\mathbf{\% o})\end{array}$ & $\begin{array}{c}\mathbf{O D} \\
(\mathbf{m L} / \mathbf{L})\end{array}$ & $\begin{array}{c}\mathbf{O D} \\
(\mathbf{\%})\end{array}$ & $\begin{array}{c}\mathbf{N O}_{\mathbf{2}}-\mathbf{N} \\
(\boldsymbol{\mu} \mathbf{m o l} / \mathbf{L})\end{array}$ & $\begin{array}{c}\mathbf{N O}_{3}-\mathbf{N} \\
(\boldsymbol{\mu} \mathbf{m o l} / \mathbf{L})\end{array}$ & $\begin{array}{c}\mathbf{P O}_{\mathbf{4}}-\mathbf{P} \\
(\boldsymbol{\mu} \mathbf{m o l} / \mathbf{L})\end{array}$ & $\begin{array}{c}\mathbf{S i O}_{\mathbf{2}} \\
(\boldsymbol{\mu} \mathbf{m o l} / \mathbf{L})\end{array}$ \\
\hline $\mathbf{9 : 0 0}$ & 0,5 & 8,16 & 29,0 & 37,70 & 3,80 & 86,99 & 0,102 & 0,054 & 1,048 & 48,166 \\
$\mathbf{1 2 : 0 0}$ & 0,5 & 8,21 & 30,0 & 37,70 & 6,61 & 153,75 & 0,040 & 0,039 & 0,963 & 42,070 \\
$\mathbf{1 5 : 0 0}$ & 0,5 & 8,15 & 30,3 & 37,70 & 7,64 & 178,55 & 0,066 & 0,313 & 1,070 & 41,503 \\
$\mathbf{1 8 : 0 0}$ & 0,4 & 8,24 & 30,2 & 38,23 & 7,71 & 180,43 & 0,117 & 0,146 & 1,238 & 47,435 \\
$\mathbf{2 1 : 0 0}$ & & 8,24 & 30,0 & 37,70 & 7,28 & 169,34 & 0,001 & 0,884 & 1,082 & 35,314 \\
$\mathbf{0 : 0 0}$ & & 8,14 & 30,0 & 37,93 & 7,62 & 177,47 & 0,001 & 0,978 & 1,201 & 48,590 \\
$\mathbf{3 : 0 0}$ & & 8,15 & 29,0 & 38,23 & 3,72 & 85,41 & 0,833 & 0,580 & 1,075 & 37,765 \\
$\mathbf{6 : 0 0}$ & & 8,01 & 28,0 & 38,23 & 2,30 & 51,96 & 0,034 & 0,929 & 1,209 & 52,682 \\
\hline
\end{tabular}

Tabela 5 - Dados de cultivo do L. vannamei.

\begin{tabular}{lcc}
\hline Discriminação & Viveiro 2 & Viveiro 3 \\
\hline Data de estocagem & $06 / 11 / 1999$ & $06 / 11 / 1999$ \\
Quantidade estocada & 25.000 & 15.000 \\
Densidade de estocagem & 5,95 & 3,57 \\
Peso médio inicial (g) & 0,004 & 0,004 \\
Biomassa inicial (g) & 100 & 60 \\
Duração do cultivo (dias) & 90 & 90 \\
Peso médio final (g) & 16,05 & 16,61 \\
Biomassa final (kg) & 192 & 130 \\
Sobrevivência (\%) & 47,80 & 52,90 \\
\hline
\end{tabular}

Trab. Oceanog. Univ. Fed. PE, Recife, v 28, n. 2, 2000. p. 209-222 

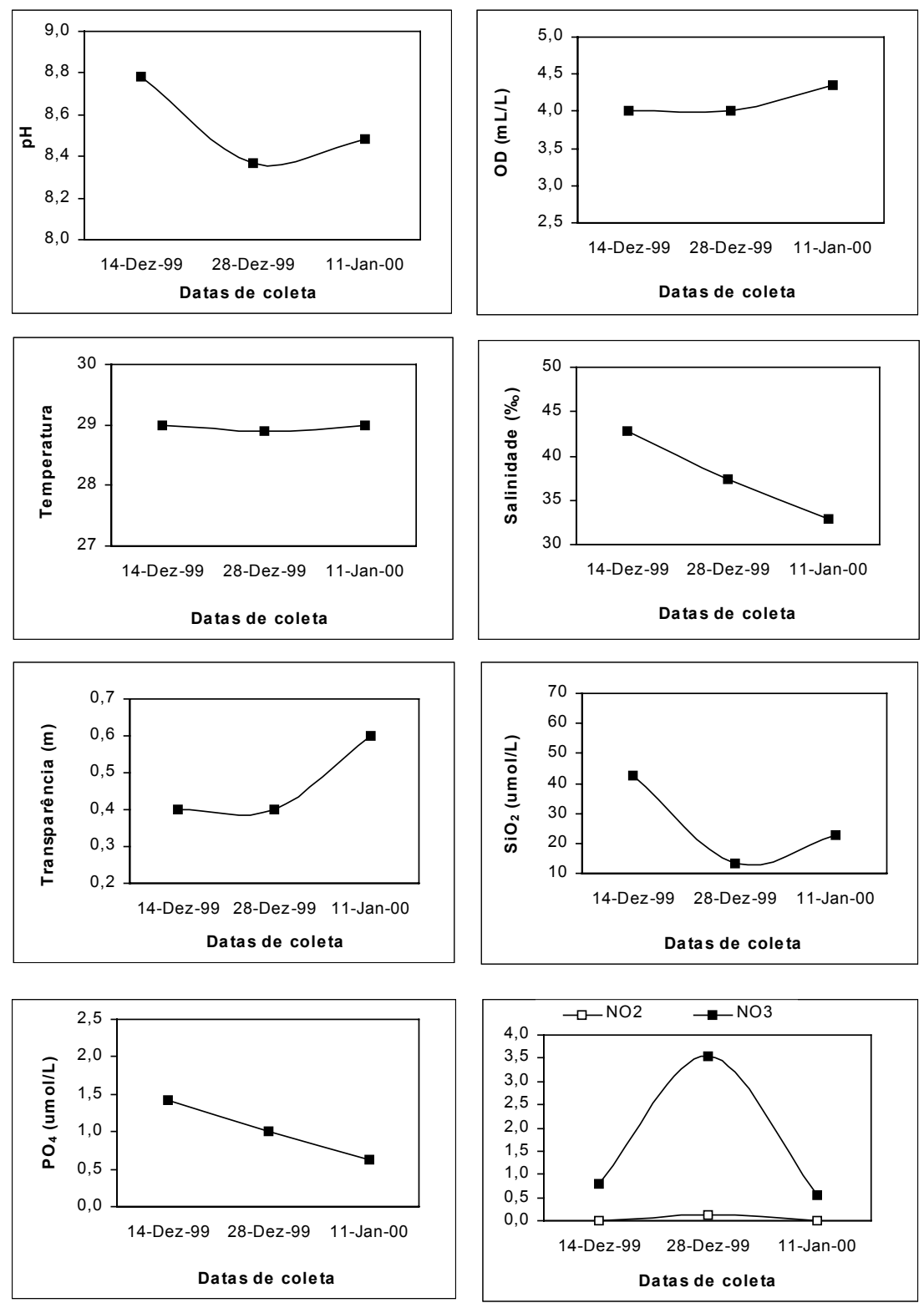

Figura 2 - Variação dos parâmetros físicos e químicos no Viveiro 2. 

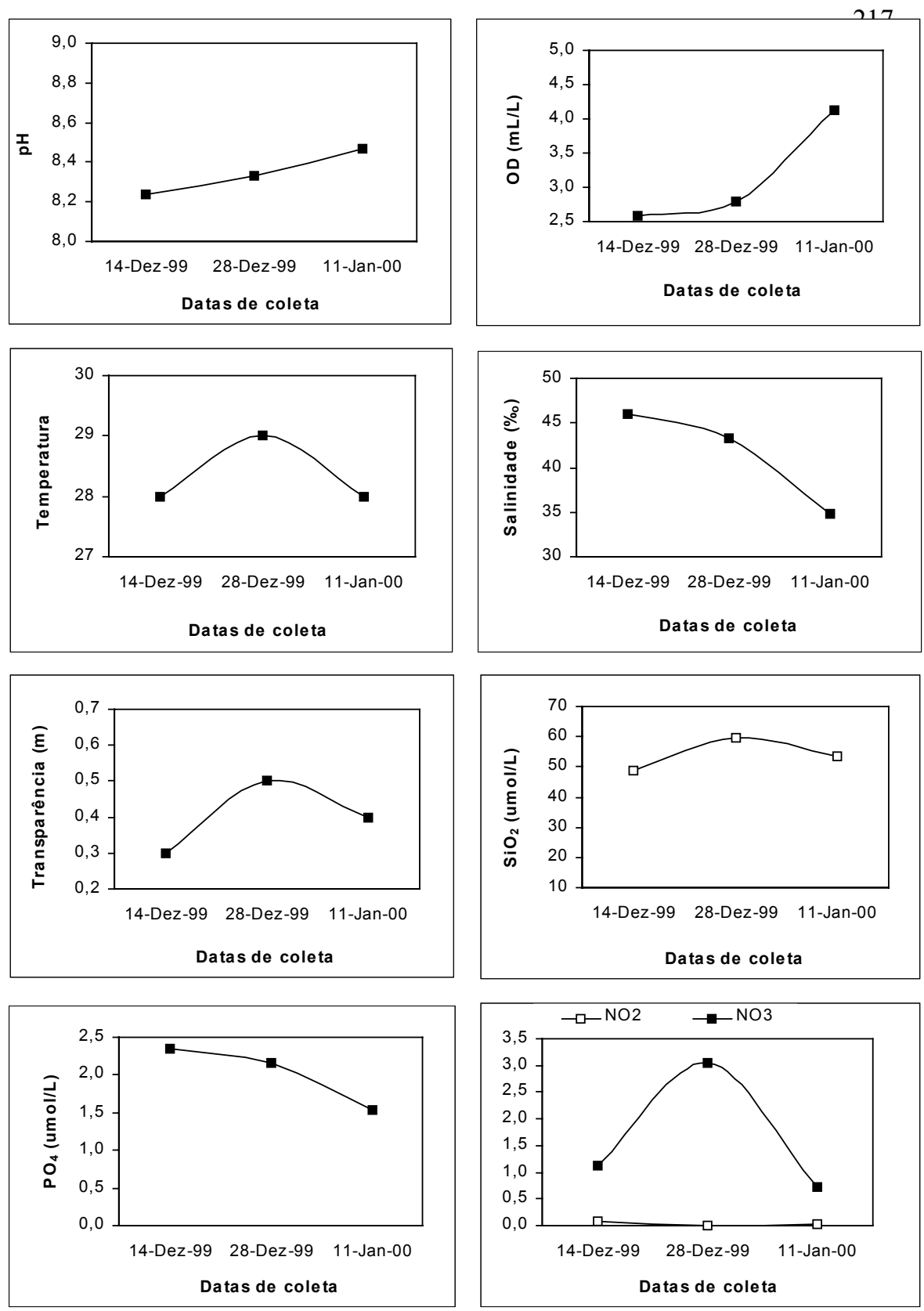

Figura 3 - Variação dos parâmetros físicos e químicos no Viveiro 3.

Trab. Oceanog. Univ. Fed. PE, Recife, v 28, n. 2, 2000. p. 209-222 

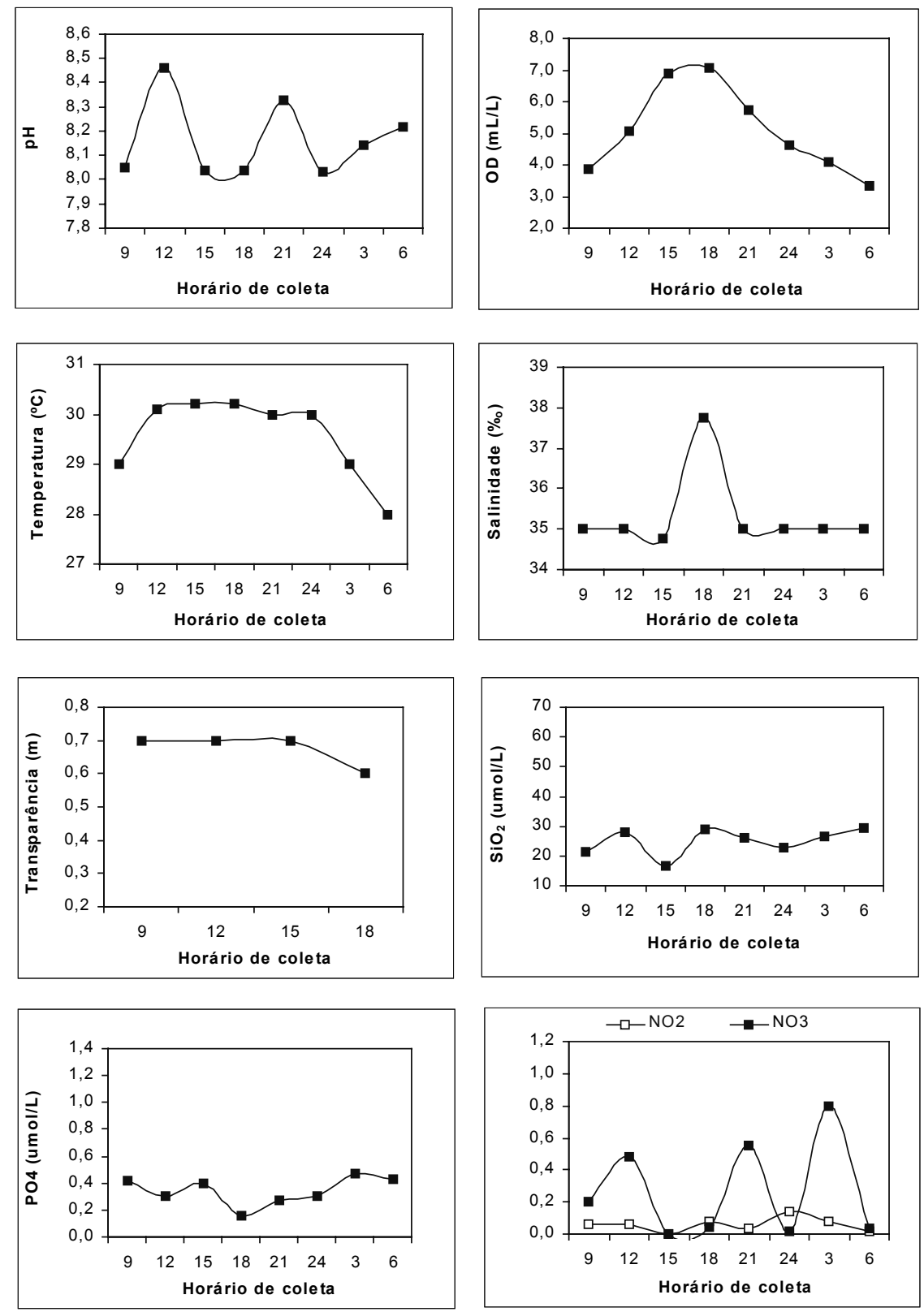

Figura 4 - Variação nictemeral dos parâmetros físicos e químicos no Viveiro 2 $(25 / 01 / 2000)$.

Trab. Oceanog. Univ. Fed. PE, Recife, v 28, n. 2, 2000. p. 209-222 

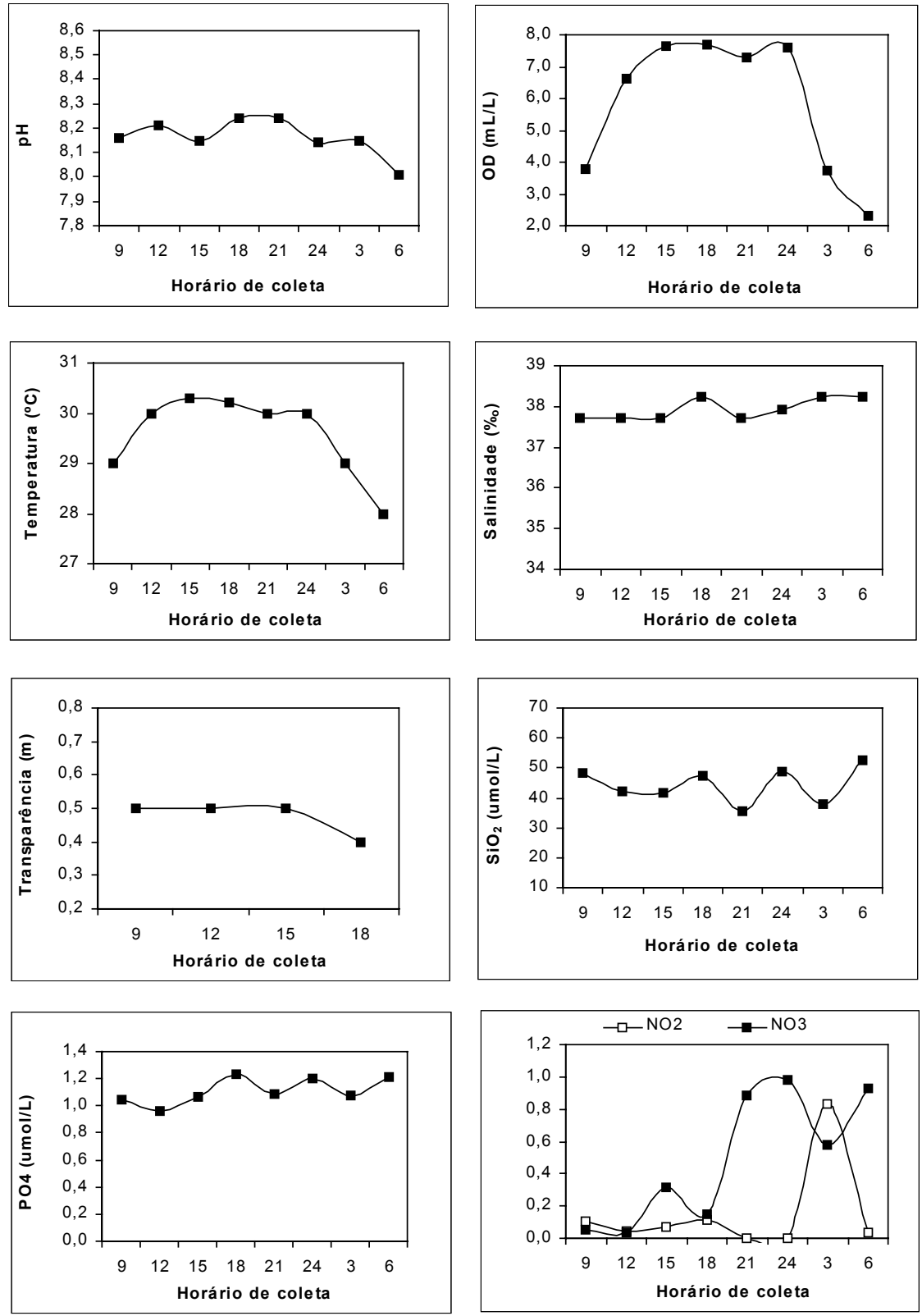

Figura 5 - Variação nictemeral dos parâmetros físicos e químicos no Viveiro $3(25 / 01 / 2000)$.

Trab. Oceanog. Univ. Fed. PE, Recife, v 28, n. 2, 2000. p. 209-222 


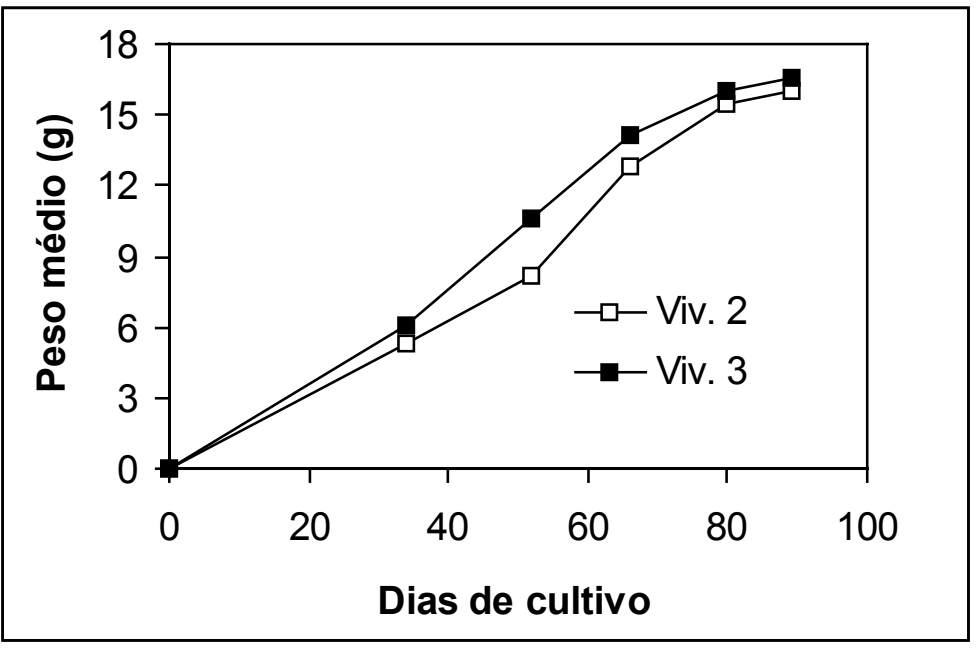

Figura 6 - Variação do peso médio de L. vannamei em função do tempo de cultivo

\section{CONCLUSÕES}

1. mesmo considerando a deficiente renovação de água em ambos os viveiros pesquisados, não foram observadas variações significativas dos parâmetros físicos e químicos;

2. os valores de salinidade, em ambos os viveiros foram algumas vezes superiores a $40 \%$ o, não evidenciando no entanto, influência negativa sobre o desempenho dos camarões. No Viv. 3, os valores foram sempre mais elevados, em razão da deficiente renovação de água;

3. o oxigênio dissolvido apresentou níveis de supersaturação em ambos os viveiros, no período diurno da variação nictemeral, indicando provavelmente, grande atividade fotossintética;

4. os valores de fosfato e silicato apresentaram-se sempre superiores no Viv. 3, em relação ao Viv. 2, inclusive durante a variação nictemeral, indicando a possibilidade de uma maior decomposição de matéria orgânica no ambiente, acumulada em função da pouca renovação de água,

Trab. Oceanog. Univ. Fed. PE, Recife, v 28, n. 2, 2000. p. 209-222 
5 o índice de produtividade do Viv. 2 foi ligeiramente superior ao do Viv. 3, em razão da maior densidade de estocagem e pelo fato de os camarões terem recebido alimentação suplementar através de ração industrializada;

6 as taxas de sobrevivência do cultivo foram relativamente baixas, podendo-se atribuir à presença de peixes predadores, que fora capturados durante a despesca e que provavelmente penetraram nos viveiros, sob a forma de larvas e alevinos;

7 o camarão L. vannamei, por sua rusticidade pode ser cultivado de forma extensiva em viveiros estuarinos, sem necessidade de aplicação de tecnologias mais sofisticadas, constituindo-se uma opção para pequenos aquicultores, que hoje já utilizam esses tipos de viveiros para cultivo de peixes, como tainhas e camorins.

\section{REFERÊNCIAS BIBLIOGRÁFICAS}

BEDIER, E.; SOYIG, C. Effects of dissolved oxygen concentration on survival and growth of Penaeus vannamei and P. stylirostris. Journal of the World Aquaculture Society, Baton Rouge, v.19, p.13-17. 1988

BOYD, C.E. Water quality management and aeration in shrimp farming. Alabama: American Soybean Association / US Wheat Associates. 1989, 70p.

BOYD.C.E. Water quality in ponds for aquaculture. Alabama Agricultural Experiment Station, Auburn University, Al., USA, 1990, 482p.

CASTRO, P. F.; MAIA, E. P.; CORREIA, E. S. Efeito da densidade de estocagem sobre o desempemho de camarão Litopenaues vannamei cultivado em sistema semi-intensivo. In: SIMPÓSIO BRASILEIRO DE AQUICULTURA, 11, 2000, Florianópolis, Anais.... Florianópolis: SIMBRAQ, 2000. Não paginado. CD-ROM.

CAVAlCANTI, L. B.; COSTA, M. L. S.; CASTRO, P. F.; CORREIA. E. S. Avaliação de cultivos do camarão marinho Litopenaeus vannamei em dois viveiros da Aquamaris Aqüicultura S.A, no período de 1996 a 1999. SIMPÓSIO BRASILEIRO DE AQUICULTURA, 11, 2000, Florianópolis, SC. Anais.... Florianópolis: SIMBRAQ, 2000. Não paginado. CD-ROM.

CLIFFORD, H. Manejo de estanques sembrados com camarón azul Litopenaeus stylirostris (Parâmetros Físico-Químicos). Panorama Aqüicola, Col. San Pedro de los Pinos, v.4, n.1, Nov/Dec, p.10-11. 1998.

Trab. Oceanog. Univ. Fed. PE, Recife, v 28, n. 2, 2000. p. 209-222 
GRASSHOF, K.; EHRHARDT, M.; KREMLING, K. (ed.) Methods of Seawater Analysis. $2^{a}$ edição, Ney York: Verlarg Chemie, 1983, 317p.

GUERRERO-GALVÁN, S. R.; PÁEZ-OSUNA, F.; RUIZ-FERNÁNDEZ, A. C.; ESPINOZA-ANGULO, R. Seasonal variation in the water quality and chlorophyll of semi-intensive shrimp ponds in a subtropical environment. Hydrobiologia, $v$. 391, p.33-45. 1999.

HOPKINS, J. S.; STOKES, A. D.; BROWDY, C. L.; SANDIFER, P. A. The relationship between feeding rate, paddle-wheel aeration rate and expected dawn dissolved oxygen in intensive shrimp ponds. Aquaculture Engineering, v.10, p.281-290. 1991.

MACEDO, S. J.; CAVALCANTI, L. B.; COSTA, K. M. P. Variação dos parâmetros físico-químicos em viveiros de cultivo da Ilha de Itamaracá (Pernambuco -Brasil). Publicação da Academia Brasileira de Ciências. Rio de Janeiro, p.73-85. 1980.

MAIA, E. P. Progresso e perspectivas da carcinicultura marinha no Brasil. In: SIMPÒSIO BRASILEIRO SOBRE CULTIVO DE CAMARÃO, 4, João Pessoa, 1993. Anais... João Pessoa: MCR, p.185-196, 1993.

MAIA, E. P.; BOLOGNA, A. S.; ARAGÃO, M. L; OLIVEIRA, A. G. Estudo preliminar sobre o cultivo super-intensivo do Litopenaues vannamei. In: SIMPÓSIO BRASILEIRO DE AQUICULTURA, 11, 2000, Florianópolis, SC. Anais.... Florianópolis: SIMBRAQ, 2000. Não paginado. CD-ROM.

NUNES, A. J. P. A critical review and update on the culture methods of the thriving business of marine shrimp farming in NE Brazil,. In: SIMPÓSIO BRASILEIRO DE AQUICULTURA, 11, 2000, Florianópolis, SC. Anais.... Florianópolis: SIMBRAQ, 2000. Não paginado. CD-ROM.

SIPAÚBA-TAVARES, L. M. Limnologia dos sistemas de cultivo. In: Valenti, W.C. (ed.). Carcinicultura de Água Doce: Tecnologia para Produção de Camarões. Brasília: IBAMA/FAPESP. p. 47-75, 1998.

STRICKLAND, J. D. H.; PARSONS, T. R. A Manual of seawater analyses.

Bulletin Fisheries Research Board of Canada, Ottawa, v.125, p.1-205, 1065.

STRICKLAND, J.D.H.; PARSONS, T.R. 1972. A pratical handbook of sea water analyses. Bulletin Fisheries Research Board of Canada, Ottawa,167, $2^{\mathrm{a}}$ Ed., $311 \mathrm{p}$

Trab. Oceanog. Univ. Fed. PE, Recife, v 28, n. 2, 2000. p. 209-222 